\title{
AVERAGE SURVIVAL ACCORDING TO THE CLINICAL STAGE IN 10,532 PATIENTS WITH BREAST CANCER TREATED BY THE BRAZILIAN UNIFIED HEALTH SYSTEM (SUS) FROM JANUARY 2011 TO DECEMBER 2019 AT PÉROLA BYINGTON HOSPITAL
}

Tayana Moreira Faria, Felipe Andreotta Cavagna¹, André Mattar¹, Jorge Yoshinori Shida', Luiz Henrique Gebrim¹ ${ }^{1}$ Hospital Pérola Byington-São Paulo (SP), Brazil.

Introduction: The distribution of the different clinical stages of cancer in the population is of paramount importance not only to assess prognosis and therapy, but mainly to establish public health strategies and priorities. The prevalence of advanced cases in some populations shows the need for public assistance policies aimed at early detection for a rapid reduction in mortality. Unfortunately, reports in Brazil about the different realities of assistance in the Brazilian Unified Health System (SUS) are scarce. Objectives: To evaluate the distribution of the different clinical stages, and the mean 5-year survival of 10,532 breast cancer patients treated by SUS at Pérola Byington Hospital, from January 2011 to December 2016. Methods: A hospital-based observational cross-sectional study was conducted. The population consisted of women with breast cancer treated by SUS at Pérola Byington Hospital. The data were registered in the institution's data collection system, based on the selection of all women diagnosed with breast cancer in the period from January 2011 to December 2016, and stratified by survival in relation to the initial clinical staging. Results: Our database consists of 620 patients in stage zero (DCIS), 2,479 patients in stage I, 3,998 in stage II, 3,082 in stage III and 353 patients in stage IV. The highest survival rate (98\%) was observed in patients with ductal carcinoma in situ. In stage I patients, the 5-year survival rate was 94.7\%. This result is slightly higher than that observed in stage II patients, where $90.6 \%$ of them had a survival of more than 5 years. In patients belonging to stages IIIA, IIIB and IIIC, the 5 -year survival was $83 \%, 72 \%$ and $57 \%$, respectively. Among the patients treated in stage IV, the 5 -year survival rate was $55 \%$. Conclusions: Among DCIS, $2 \%$ had recurrence and death within 5 years. Survival in patients belonging to stages I and II was $94.7 \%$ and $90.6 \%$, respectively, which is very close, according to literature data. The survival of patients belonging to stages IIIA and IIIB exceeded 70\%, indirectly showing the efficacy of locoregional and systemic therapy at SUS, which applies to stages IIIC and IV. These reports are unprecedented in the database of the Unified Health System of the Municipality of São Paulo, in a population that undergoes only opportunistic screening. 